\title{
Index des personnes et des lieux
}

Aitzing, Michael 103

Aix-en-Provence 128

Alençon 131

Allonne 131

Amboise 8, 18, 20, 34, 53, 57-59, 62, 85,

124-125, 127-128, 130, 132

Angers 44, 132

Anvers 84, 99-100, 102, 105, 118

Ardennes 131

Aubigné, Agrippa d' 86, 96

Augsbourg 102, 118

Auvergne 124, 129

Auxerre 96

Avignon 33

Bassigny 131

Begon, Michel 106

Belon, Pierre 34

Benoist, René 41-43, 47-49

Berthelin, Madeleine 136, 144

Beys, Gilles 65

Bèze, Théodore de 19, 24, 28, 34, 52

Biron, Charles de Gontaut, baron de 118

Blaye 127

Blois 106

Bodin, Jean 7

Bontemps, Léger de 136-138

Bordeaux 125, 127

Bordeaux, Christophe de 135-145

Bordeaux, Jean de 135

Bourbon, Antoine de, roi de Navarre 95, 138

Bourg, Anne du 54, 76, 88, 111-114, 119

Bourges 34

Bourgogne 93

Bourjac, Félix 29

Brantôme, Pierre de Bourdeille, seigneur de 61,77

Braun, George 105, 116

Breughel, Pieter l'Ancien 89

Brigard, François 64

Brossat, Alain 85

Caen 25,26

Cahors 87-89, 96

Calais $56,61,78,117$

Calvin, Jean 45-46, 123, 137, 139

Carcassonne 30, 126, 132

Carentan 131
Caron, Antoine 93

Casimir, Jean de Bavière 99, 115

Castagna, Giovanni Battista, archevêque de

Rossano 41

Castellin, Jean 84

Cateau-Cambrésis 52, 57

Catherine de Médicis 25, 34, 40, 58, 74, 93

Caudebec 131

Chalon-sur-Saône 54, 124-125

Champagne 131

Charlemagne 32

Charles IX, roi de France 58-60, 63-64, 74-75, 93, 96

Chartier, Jehan 128

Chartier, Roger 13, 136

Chartres 135, 140, 144

Chastel, Jehan 128

Châtillon, Gaspard de, amiral de Coligny 63, 139

Chaumont 131

Chesneau, Nicolas 39, 43-47

Clermont 124, 126-127, 129-130

Cock, Hieronymus 99

Cologne 98-106, 110, 116, 118

Compiègne 57,58

Conches 131

Condé, Louis $1^{\text {er }}$ de Bourbon, prince de 28-31, 34-35, 59-61, 78, 94, 131, 135, 139,144

Crespin, Jean 88, 95

Dathie, Pierre 125

Dauphiné 31, 56, 62

Des Adrets, François de Beaumont, baron 31, 33, 86

Des Autels, Guillaume 59

Désiré, Artus 137-138, 143-144

Dijon 123, 126, 129, 132, 136

Diois 29

Dreux 61

Dubois, François 96

Düsseldorf 105, 118

Duval, Antoine 45-46

Eco, Umberto 37

Écosse 54, 60-61, 66, 92

Écouen 58

Ehrmann, Jean 93

Estienne, Robert 68 
Francfort $65,98,104$

François I ${ }^{\text {er }} 77,142$

François II 8, 18, 51-52, 54-55, 59, 6164, 93

Frémy, Claude 45

Fumée, Anthoine 126

Gaignières, Roger de 69

Gaillac 124, 132

Gairard, Anthoine 127

Gay, Thomas 143

Genève $25,52,57,74,83,84,118,137$

Ghirlandaio, Domenico 89

Gibier, Éloi 24-25, 29

Gilmont, Jean-François 25

Gisors 131

Godion de Lestang, Alexandre 26

Goody, Jack 12

Granger, Hélye 124

Grangier, Michel 25

Grenoble 30

Guise, Claude $1^{\text {er }}$ de Lorraine, duc de 66

Guise, François de Lorraine, duc de 29-

$31,51-63,66,71-74,82-83,85,92,91-$

$97,114,139,141-142$

Guise, Henri de Lorraine, duc de 8

Guise, Louis de Lorraine, cardinal de 8, 92, 94

Guise, maison de 8, 19-20, 35, 44, 51, 54, $72,77,92-93,131$

Habermas, Jürgen 40

Hans, Jean de 142-143

Haton, Claude 61-62

Heidelberg 25

Hémard, Robert 94

Henri II, roi de France $7,40,51-52,55-$

$56,58,61-62,64,73,78,88,114,142$

Henri III, Henri d'Anjou, puis roi de

France 116, 119

Henri IV, Henri de Navarre 116-119, 124

Hervet, Gentian 41-42, 45-47, 60

Hogenberg, Franz 92, 98-120

Hollywood, John de 70

Hotman, François 19, 52-54

Innis, Harold 12

Jarnac 140

Jazeneuil 140

Joubert, Laurent 68

Kittler, Friedrich 13
Langres 127

Languedoc 30-31

La Noue, François de 82, 86

La Planche, Louis Régnier, sieur de, 77

La Ramée, Pierre de 67

La Rochefoucauld, François, duc de 139

Lasswell, Harold D. 15

Le Maçon, Jean 27

Le Mans 29

Le Normant, Jehan 127

Le Picart, François 43-44, 60

Le Prestre, Marie 65

L'Espine, Jean de 47-49

L'Estoile, Pierre de 65, 77

Letellier, Nicolas 124

Leunclavius, Johannes 65

Le Vignon, Pierre 84

L'Hospital, Michel de 8, 19, 72

Lindan, Guillaume 45-46

Lisieux 123

Loches 58

Lomar, Agnes 101

Lorges, Gabriel de 139

Lorraine, Charles, cardinal de 42, 44-46, $73,76,92,114$

Lorraine, René, marquis d'Elbeuf 66

Lorraine, Renée de 44

Louis $\mathrm{I}^{\text {er }}$, le Pieux 32

Louis $1^{\text {er }}$, prince de Bourbon-Condé 82, 86

Luhmann, Niklas 12-17

Lyon 25, 32-33, 124, 128, 132, 137

Mâcon 123, 127-128

Marot, Clément 79, 137

Martel, Geoffroy 140

Marx, Karl 129

McLuhan, Marshall 12

Meaux 28, 85, 124

Mercator, Gerhard 105

Merleau-Ponty, Maurice 123

Metz 56

Millet, Jehan 125

Miron, François 64

Miron, Marc 64

Moissac 126, 127

Moncontour 140

Montélimar 31

Montferrand 124

Montgommery, Gabriel de Lorges, compte de 32

Montmorency, Anne de 34, 55, 60

Moreaux, Isabel 128

Morel, Léonard 90 
Moyen-Orient 38

Nantes 8, 116, 119

Naples 55

Neux 64

Nîmes 30-31, 85

Normandie 32, 131

Notre-Dame-de-Cléry 61

Novellanus, Simon 103

Obry, Nicole 77

Orléans 24-29, 34, 60-63, 72, 78, 83, 94, 96

Ortelius, Abraham 99, 105

Paré, Ambroise 64

Paris 29, 37, 39, 45, 51, 64, 67, 82, 88, 124, 136, 138-140, 143

Pasquier, Étienne 85

Pays-Bas 116

Perrissin, Jacques $83-84,87,89-90,94-$ 95, 98-99

Picardie 131

Pierrelatte 86

Plantijn, Christoffel 99, 105

Platon 10, 38

Poissy 61

Poitiers 26

Poltrot de Méré, Jean 30, 55, 57, 62-63, $72,96,139,141$

Pont-Audemer 131

Porchier, François 123

Porcy 124

Prévost, Mathurin 65

Provence 33, 56, 86, 126

Psaume, Nicolas, évêque de Verdun 61

Rasse des Neux, François 64-79

Ravaillac, Jacques 118

Reims 44, 60-61, 73

Riom 129

Rome 35, 73

Romorantin 57-58
Rouen 28-29, 124

Rousseau, Pierre 44

Rousseau, Ursula 135

Saint-André 61

Saint-Denis 137, 140

Saint-Germain-en-Laye 8, 25, 124,

130

Saint-Laurent 44

Saint-Marcellin 86

Schlögl, Rudolf 14, 16

Senlis 131

Sens 32, 85, 93-94, 96

Sermisy, Claudin de 137

Sisteron 33

Spifame, Jacques 24

Stempel, Gerhard 105

Strasbourg 52, 54, 57

Strozzi, Laurent, évêque d'Albi 33

Thou, Christophe de 25

Tortorel, Jean 83-84, 87, 89-90, 94-95, 98-99

Toulouse 30, 127-129

Touraine 132

Tours $25,85,92,94-96,125,132$

Trente $40-42,45$

Troyes 125

Val, Denys du 65

Valence 86

Valentinois 29

Van Meteren, Emmanuel 103

Viart, Jacques 126

Vierger, Gilles du 132

Vietnam 84

Vire 131

Wassy 26-29, 35, 52, 55, 60-61, 82-86, 89-96

Watt, Ian 12

Wechel, André 65

Weinsberg, Hermann 104 
